\title{
CODIMENSION ONE SYMPLECTIC FOLIATIONS AND REGULAR POISSON STRUCTURES
}

\author{
VICTOR GUILLEMIN, EVA MIRANDA, AND ANA RITA PIRES \\ Dedicated to the memory of Paulette Libermann whose cosymplectic manifolds \\ play a fundamental role in this paper.
}

\begin{abstract}
In this short note we give a complete characterization of a certain class of compact corank one Poisson manifolds, those equipped with a closed one-form defining the symplectic foliation and a closed two-form extending the symplectic form on each leaf. If such a manifold has a compact leaf, then all the leaves are compact, and furthermore the manifold is a mapping torus of a compact leaf.

These manifolds and their regular Poisson structures admit an extension as the critical hypersurface of a Poisson $b$-manifold as we will see in GMP.
\end{abstract}

\section{INTRODUCTION}

Given a regular Poisson structure we have an associated symplectic foliation $\mathcal{F}$ given by the distribution of Hamiltonian vector fields. In this short paper we study some properties of codimension one symplectic foliations for regular Poisson manifolds and define some invariants associated to them.

In Section 2.1 we introduce the first invariant, associated to the defining one-form of the foliation. We will see in Section 3.1 that this invariant measures how far a Poisson manifold is from being unimodular. When this invariant vanishes we can choose a defining one-form for the symplectic foliation which is closed. In particular when this invariant

Date: May 31, 2018.

Eva Miranda is partly supported by the DGICYT/FEDER project MTM200907594: Estructuras Geometricas: Deformaciones, Singularidades y Geometria Integral. Ana Rita Pires was partly supported by a grant SFRH / BD / 21657 / 2005 of Fundação para a Ciência e Tecnologia. 
vanishes, the Godbillon-Vey class of the foliation vanishes too (as had been previously observed by Weinstein in [We] $)$.

In Section 2.2, we introduce another invariant also related to the global geometry of these manifolds. The second invariant measures the obstruction to the existence of a closed two-form on the manifold that restricts to the symplectic structure on each leaf. This invariant had been previously studied by Gotay in [Go in the setting of coisotropic embeddings. This invariant also has a nice interpretation for symplectic fiber bundles (see GLSW]).

In section 3.2, we explore some of the global implications of the vanishing of these two invariants for compact manifolds. In particular we show that if they vanish and the foliation $\mathcal{F}$ has at least one compact leaf $L$, then all leaves are compact and $M$ itself is the mapping torus associated with the holonomy map of $L$ onto itself. (In particular, the leaves of $\mathcal{F}$ are the fibers of a fibration of $M$ over $\mathbb{S}^{1}$.)

In Section 3.3, we give the Poisson version of this mapping torus theorem. These manifolds turn out to be cosymplectic manifolds in the sense of Libermann $[\mathrm{L}]$.

We also briefly describe another global consequence of the vanishing of these invariants: A $2 n$-dimensional Poisson manifold $(M, \Pi)$, is a Poisson $b$-manifold if the section $\Pi^{n}$ of $\Lambda^{n}(M)$ intersects the zero section of this bundle transversally. For such manifolds it is easy to see that this intersection is a regular Poisson manifold with codimension one symplectic leaves and that its first and second invariants vanish. A much harder result (which will be the topic of a sequel to this paper) is that the converse is also true.

As an application of theorem[19 we give an explicit description of the Weinstein's groupoid integrating the Poisson structure of these regular manifolds in the case there is a compact leaf. We do it in section 3.4.

The main result of this paper (Theorem 19) is related to previous results of HongJun Li [Li] where a symplectic version of Tischler's theorem [Ti] for cosymplectic manifolds is given. Tischler's theorem asserts that a compact manifold that admits a closed one-form is a fibration over a circle, but not that this fibration is the one given by the prescribed closed one-form. Li's main theorem in [Li] asserts that cosymplectic manifolds are equivalent to symplectic mapping tori. The main

\footnotetext{
${ }^{1}$ A cosymplectic manifold is manifold $M^{2 n+1}$ together with a closed one-form $\eta$ and a closed two-form, $\omega$ such that $\eta \wedge \omega^{n}$ is a volume form.
} 
difference with our result is that we work precisely with the foliation given by the closed one-form (whose existence is guaranteed by the vanishing of the first invariant) and with the Poisson structure guaranteed by the vanishing of the second invariant. The construction provided by Tischler and Li for this foliation is purely topological and does not apply to the foliation given by the closed one-form.

Theorems 19 and 21 relate to the work of Mélanie Bertelson in [B] because they provide natural examples of regular foliated manifolds that admit a Poisson structure. In [B], Bertelson studies topological obstructions for a foliated manifold to admit regular Poisson structures and uses the h-principle in the Poisson context to construct examples of such structures. Our objects with vanishing first obstruction class may provide examples of application of Bertelson's results. In particular, these results ensure that the vanishing of just the first invariant, together with some additional conditions related to the defining one-form that it is associated to, implies that there exists a Poisson structure.

Acknowledgements We are grateful to the referees of this paper for their useful comments and for drawing our attention to many interesting papers in the literature on related matters. The second author wishes to thank the organizers of the Conference Poisson 2010 (where part of these results where presented) for their hospitality and a perfect working atmosphere. Some of the results in this paper were improved thanks to conversations with Rui Loja Fernandes and David Martínez Torres. In particular, we are thankful to David Martínez Torres for useful observations about the symplectic groupoids integrating these manifolds, for a different interpretation of the unimodularity condition of Theorem 10, and for suggesting Example 22, and to Rui Loja Fernandes for pointing out the connection of the vanishing of our second invariant with the results in his paper with Marius Crainic [CF].

\section{INTRODUCING TWO INVARIANTS OF A FOLIATION}

2.1. The defining one-form of a foliation and the first obstruction class. Let $M$ be a real manifold, $\mathcal{F}$ a transversally orientable codimension one foliation of $M$, and write $\Omega(M)$ simply as $\Omega$.

Definition 1. A form $\alpha \in \Omega^{1}$ is a defining one-form of the foliation $\mathcal{F}$ if it is nowhere vanishing and $i_{L}^{*} \alpha=0$ for all leaves $L \stackrel{i_{L}}{\hookrightarrow} M$.

A basic property of this defining one-form is: 
Lemma 2. For $\mu \in \Omega^{k}$, we have $\mu \in \alpha \wedge \Omega^{k-1}$ if and only if $i_{L}^{*} \mu=0$ for all $L \in \mathcal{F}$. have

In particular, because $i_{L}^{*}(d \alpha)=d\left(i_{L}^{*} \alpha\right)=0$ for all leaves $L \in \mathcal{F}$, we

$$
d \alpha=\beta \wedge \alpha \text { for some } \beta \in \Omega^{1} .
$$

Thus, $\Omega_{1}=\alpha \wedge \Omega$ is a subcomplex of $\Omega$ and $\Omega_{3}=\Omega / \alpha \wedge \Omega$ a quotient complex. Although the spaces $\Omega_{1}$ and $\Omega_{3}$ are defined using $\alpha$, they do not depend on the particular choice of the defining one-form. Indeed, let $\alpha$ and $\alpha^{\prime}$ be distinct defining one-forms for the foliation $\mathcal{F}$, then we must have $\alpha^{\prime}=f \alpha$ for some nonvanishing $f \in C^{\infty}(M)$, thus producing the same complexes $\Omega_{1}$ and $\Omega_{3}$. The statement of Lemma 2 simply gives a necessary and sufficient condition for a $k$-form $\mu \in \Omega^{k}$ to be in $\Omega_{1}^{k}$.

Writing $\Omega_{2}=\Omega$ we have a short exact sequence of complexes

$$
0 \longrightarrow \Omega_{1} \longrightarrow \Omega_{2} \stackrel{j}{\longrightarrow} \Omega_{3} \longrightarrow 0 .
$$

Note that although the form $\beta$ is not unique for a fixed choice of $\alpha$, the projection $j \beta$ is: two distinct forms $\beta$ and $\beta^{\prime}$ must differ by a multiple of $\alpha$ (by a function). From (1) we get $0=d(d \alpha)=d \beta \wedge \alpha-$ $\beta \wedge \beta \wedge \alpha=d \beta \wedge \alpha$, that is, $\alpha$ and $d \beta$ are dependent, so we must have $d \beta \in \Omega_{1}$. Then, $d(j \beta)=0$ and we can define the first obstruction class $c_{\mathcal{F}} \in H^{1}\left(\Omega_{3}\right)$ to be

$$
c_{\mathcal{F}}=[j \beta] .
$$

Proposition 3. The first obstruction class $c_{\mathcal{F}}$ does not depend on the choice of the defining one-form $\alpha$.

Proof. Let $\alpha$ and $\alpha^{\prime}$ be distinct defining one-forms for the foliation $\mathcal{F}$, then $\alpha^{\prime}=f \alpha$ for some nonvanishing $f \in C^{\infty}(M)$. We have

$$
d \alpha^{\prime}=d f \wedge \alpha+f d \alpha=d f \wedge \alpha+f \beta \wedge \alpha=\left(\frac{d f}{f}+\beta\right) \wedge \alpha^{\prime},
$$

so $\beta^{\prime}=d(\log |f|)+\beta$. Thus $\left[j \beta^{\prime}\right]=[j \beta]$.

Theorem 4. The first obstruction class $c_{\mathcal{F}}$ vanishes identically if and only if we can chose $\alpha$ the defining one-form of the foliation $\mathcal{F}$ to be closed. 
Proof. The first obstruction class $c_{\mathcal{F}}=[j \beta]$ vanishes identically if and only if $\beta=d f+g \alpha$ for some $f, g \in C^{\infty}(M)$. Replacing $\alpha$ by $\alpha^{\prime}=e^{-f} \alpha$ we get

$$
\begin{aligned}
d\left(e^{-f} \alpha\right) & =-e^{-f} d f \wedge \alpha+e^{-f} d \alpha \\
& =-e^{-f} d f \wedge \alpha+e^{-f} \beta \wedge \alpha \\
& =-e^{-f} d f \wedge \alpha+e^{-f} d f \wedge \alpha+e^{-f} g \alpha \wedge \alpha \\
& =0 .
\end{aligned}
$$

\subsection{The defining two-form of a foliation and the second ob-} struction class. Assume now that $M$ is endowed with a regular corank one Poisson structure $\Pi$ and that $\mathcal{F}$ is the corresponding foliation of $M$ by symplectic leaves. Furthermore, assume that the first obstruction class $c_{\mathcal{F}}$ vanishes and therefore by Theorem 4 the foliation is defined by a closed one-form $\alpha$. Later, in Theorem 10, we will show that this happens exactly when the foliation $\mathcal{F}$ is unimodular.

Definition 5. A form $\omega \in \Omega^{2}$ is a defining two-form of the foliation $\mathcal{F}$ induced by the Poisson structure $\Pi$ if $i_{L}^{*} \omega=\omega_{L}$ is the symplectic form on each leaf $L \stackrel{i_{L}}{\hookrightarrow} M$.

Using Lemma 2 again, and because $i_{L}^{*}(d \omega)=d\left(i_{L}^{*} \omega\right)=d \omega_{L}=0$ for all leaves $L \in \mathcal{F}$, we have

$$
d \omega=\mu \wedge \alpha \text { for some } \mu \in \Omega^{2} .
$$

Again note that although the form $\mu$ is not unique for fixed choices of $\alpha$ and $\omega$, the projection $j \mu$ is. From (2) and $\alpha$ being closed, we conclude that $d \mu \wedge \alpha=0$, that is $\alpha$ and $d \mu$ are dependent, and so $d \mu \in \Omega_{1}$. Then, $d(j \mu)=0$ and we can define the second obstruction class $\sigma_{\mathcal{F}} \in H^{2}\left(\Omega_{3}\right)$ to be

$$
\sigma_{\mathcal{F}}=[j \mu] .
$$

Proposition 6. The second obstruction class $\sigma_{\mathcal{F}}$ does not depend on the choice of the defining two-form $\omega$.

Proof. Let $\omega$ and $\omega^{\prime}$ be distinct defining two-forms for the foliation $\mathcal{F}$. We must have $\omega^{\prime}=\omega+\nu$ for some $\nu \in \Omega^{2}$ such that $i_{L}^{*} \nu=0$ for every leaf $L \in \mathcal{F}$. Then, by Lemma 2, $\nu=\alpha \wedge \xi$ for some $\xi \in \Omega^{1}$ and we 
have:

$$
\begin{aligned}
d \omega^{\prime} & =d \omega+d \nu \\
& =\mu \wedge \alpha-\alpha \wedge d \xi \\
& =(\mu+d \xi) \wedge \alpha .
\end{aligned}
$$

Thus, $\mu^{\prime}=\mu+d \xi$ and $\left[j \mu^{\prime}\right]=[j \mu]$.

Theorem 7. The second obstruction class $\sigma_{\mathcal{F}}$ vanishes identically if and only if we can chose $\omega$ the defining two-form of the foliation $\mathcal{F}$ to be closed.

Proof. The second obstruction class $\sigma_{\mathcal{F}}=[j \mu]$ vanishes identically if and only if $\mu=d \nu+\gamma \wedge \alpha$ for some $\nu, \gamma \in \Omega^{1}$. In this case, we have $d \omega=d \nu \wedge \alpha$, and so the two-form $\omega^{\prime}=\omega-\nu \wedge \alpha$ will be closed, with its restriction to an arbitrary leaf $L$ still being $\omega_{L}$. Conversely, if $\omega$ is closed, then $\mu \wedge \alpha=d \omega=0$, the forms $\mu$ and $\alpha$ are dependent, and $[j \mu]=0$.

We will see in Section 3 that under certain conditions, a foliation on $M$ with vanishing first and second invariants is in fact a fibration, and in that case we can re-interpret Theorem 7 above in the light of Theorem 1 in GLSW, as characterizing the extension of a closed twoform on a fiber to a closed two-form on the total manifold.

\subsection{The modular vector field and modular class of a Poisson}

manifold. We follow Weinstein [We for the description of modular vector field and the modular class of a Poisson manifold. A complete presentation of these can also be found in [Ko].

The modular vector field of a Poisson manifold measures how far Hamiltonian vector fields are from preserving a given volume form. A simple example is that of a symplectic manifold endowed with the volume form that is the top power of its symplectic form: the modular vector field will be zero because this volume form is invariant under the flow of any Hamiltonian vector field.

Definition 8. Let $(M, \Pi)$ be a Poisson manifold and $\Theta$ a volume form on it, and denote by $u_{f}$ the Hamiltonian vector field associated to a smooth function $f$ on $M$. The modular vector field $X_{\Pi}^{\Theta}$ is the derivation given by the mapping

$$
f \mapsto \frac{\mathcal{L}_{u_{f}} \Theta}{\Theta} .
$$


When both the Poisson structure and the volume form on $M$ are implicit, we denote the modular vector field by $v_{\text {mod }}$.

The modular vector field has the following properties [We]:

(1) The flow of the modular vector field preserves the volume and the Poisson structure:

$$
\mathcal{L}_{X_{\Pi}^{\Theta}}(\Pi)=0, \quad \mathcal{L}_{X_{\Pi}^{\Theta}}(\Theta)=0
$$

(2) When we change the volume form, the modular vector field changes by:

$$
X_{\Pi}^{H \Theta}=X_{\Pi}^{\Theta}-u_{\log (H)}
$$

where $H \in C^{\infty}(M)$ is nonvaninshing and positive (we are assuming that our manifold is oriented and transversally oriented). This implies that the class of the modular vector field in the first Poisson cohomology group is independent of the volume form chosen. This class is called the modular class of the Poisson manifold. A Poisson manifold is called unimodular if this cohomology class is zero. As remarked above, symplectic manifolds are unimodular.

(3) If $M$ is 2-dimensional with Poisson structure given by the bracket $\{x, y\}=f(x, y)$ and volume form $\Theta=d x \wedge d y$, then the modular vector field is $u_{f}$, the Hamiltonian vector field associated to $f$ with respect to the standard Poisson structure $\Theta^{\sharp}$. In particular, the modular vector field is tangent to the zero level set of $f$.

\section{The CASE WITH VANISHING INVARIANTS}

3.1. Vanishing first invariant: unimodular Poisson manifolds. Assume again that $\left(M^{2 n+1}, \Pi\right)$ is a corank one Poisson manifold and $\mathcal{F}$ is its foliation by symplectic leaves. Fix $\alpha \in \Omega^{1}(M)$ and $\omega \in \Omega^{2}(M)$ defining one- and two-forms of the foliation, respectively. Let us compute the modular vector field associated to the volume form $\Theta=\alpha \wedge \omega^{n}$ :

$$
\begin{aligned}
\mathcal{L}_{u_{f}} \Theta & =d\left(\iota_{u_{f}} \Theta\right) \\
& =d\left(\iota_{u_{f}} \alpha \wedge \omega^{n}\right) \\
& =-n d\left(\alpha \wedge d f \wedge \omega^{n-1}\right) \\
& =n \alpha \wedge d f \wedge \beta \wedge \omega^{n-1},
\end{aligned}
$$

where $\beta \in \Omega^{1}(M)$ is such that $d \alpha=\alpha \wedge \beta$. On the last step we use the fact that $d \omega \wedge \alpha=0$, from equation 2 , 
By definition of modular vector field, we have

$$
n \alpha \wedge d f \wedge \beta \wedge \omega^{n-1}=\left(\iota_{v_{\text {mod }}} d f\right) \alpha \wedge \omega^{n} .
$$

Furthermore, $v_{\text {mod }}$ is tangent to the leaves $L$ of $\mathcal{F} 2$ so (3) implies that

$$
n d f_{L} \wedge \beta_{L} \wedge \omega_{L}^{n-1}=\left(\iota_{v_{\bmod }} d f_{L}\right) \omega_{L}^{n},
$$

where as before $\omega_{L}=i_{L}^{*} \omega$, and similarly $f_{L}=i_{L}^{*} f$ and $\beta_{L}=i_{L}^{*} \beta$.

On the other hand, because $\left(d f_{L} \wedge \omega_{L}^{n}\right)$ is a $(2 n+1)$-form on a $(2 n)$ dimensional manifold $L$, we have

$$
\iota_{v_{\text {mod }}}\left(d f_{L} \wedge \omega_{L}^{n}\right)=0
$$

and hence

$$
\left(\iota_{v_{\text {mod }}} d f_{L}\right) \omega_{L}^{n}=d f_{L} \wedge n \iota_{v_{\text {mod }}} \omega_{L} \wedge \omega_{L}^{n-1}
$$

From (4) and (5) we conclude that for all $f_{L} \in C^{\infty}(L)$,

$$
\omega_{L}^{n-1} \wedge d f_{L} \wedge\left(\iota_{v_{\bmod }} \omega_{L}-\beta_{L}\right)=0 .
$$

This, together with the fact that for a symplectic form $\omega$ and a two-form $\mu$, we have $\omega^{n-1} \wedge \mu=0$ if and only if $\mu=0$, implies the following:

Theorem 9. Consider the Poisson manifold $\left(M^{2 n+1}, \Pi\right)$ with volume form $\Theta=\alpha \wedge \omega^{n}$, where $\alpha$ and $\omega$ are defining one- and two-forms of the induced foliation.

Then, the modular vector field is the vector field which on every symplectic leaf $L \in \mathcal{F}$ satisfies

$$
\iota_{v_{\text {mod }}} \omega_{L}=\beta_{L},
$$

where $\beta_{L}=i_{L}^{*} \beta$ is such that $d \alpha=\beta \wedge \alpha$.

A corollary of Theorem 9 is the following criterion for unimodularity 3 of corank one Poisson manifolds:

Theorem 10. A corank one Poisson manifold $(M, \Pi)$ with induced symplectic foliation $\mathcal{F}$ is unimodular if and only if the first obstruction class of the foliation $c_{\mathcal{F}}$ vanishes identically.

\footnotetext{
${ }^{2}$ See for instance section 5 in $\mathrm{We}$.

${ }^{3}$ We thank David Martínez-Torres for pointing out that an alternative proof of this can be obtained via formula (4.6) in [KLW]: this alternative proof requires the use of the interpretation of the modular class as one-dimensional representations, in what seems to be an interesting but longer path. See also [ELW].
} 
Proof. Recall that $(M, \Pi)$ is unimodular if and only if there exists a volume form on it such that the corresponding modular vector field is zero. Also, recall from Theorem 4 that $c_{\mathcal{F}}$ vanishes identically if and only if there exists a closed defining one-form $\alpha$.

But by Theorem 9, the modular vector field $v_{\bmod }=X_{\Pi}^{\alpha \wedge \omega^{n}}$ is zero if and only if $\beta_{L}=i_{L}^{*} \beta=0$ for every leaf $L \in \mathcal{F}$, which by Lemma 2 is equivalent to $\beta \in \alpha \wedge \Omega$, thus making $\alpha$ closed since $d \alpha=\beta \wedge \alpha$.

Remark 11. Given a transversally orientable foliation with defining one-form $\alpha$, the Godbillon-Vey class is defined as the class of the 3-form $\beta \wedge d \beta$. From Theorem 10 we deduce in particular that for unimodular Poisson manifolds this 3 -form vanishes. The converse is not true as observed by Weinstein in [We].

3.2. Vanishing first invariant: a topological result. We begin by recalling Reeb's global stability theorem about codimension one foliations:

Theorem 12. $\mathrm{Re}$ Let $\mathcal{F}$ be a transversely orientable codimension one foliation of a compact connected manifold $M$. If $\mathcal{F}$ contains a compact leaf $L$ with finite fundamental group, then every leaf of $\mathcal{F}$ is diffeomorphic to $L$.

Furthermore, $M$ is the total space of a fibration $f: M \rightarrow \mathbb{S}^{1}$ with fiber $L$, and $\mathcal{F}$ is the fiber foliation $\left\{f^{-1}(\theta) \mid \theta \in \mathbb{S}^{1}\right\}$.

The key point in the proof of Theorem 12 is that such a foliation with a compact leaf has trivial holonomy (see for example [CN]). Since a foliation defined by a closed one-form has trivial holonomy as well (see again [CN], p.80), the proof and conclusions of Reeb's theorem hold in the following case:

Theorem 13. Let $\mathcal{F}$ be a transversely orientable codimension one foliation of a compact connected manifold $M$ with $c_{\mathcal{F}}=0$. If $\mathcal{F}$ contains a compact leaf $L$, then every leaf of $\mathcal{F}$ is diffeomorphic to $L$.

Furthermore, $M$ is the total space of a fibration $f: M \rightarrow \mathbb{S}^{1}$ with fiber $L$, and $\mathcal{F}$ is the fiber foliation $\left\{f^{-1}(\theta) \mid \theta \in \mathbb{S}^{1}\right\}$.

Remark 14. A theorem of Tischler [Ti] says that a compact manifold endowed with a non-vanishing closed one-form must be a fibration over a circle. However, this fibration need not coincide with the codimension one foliation defined by the closed one-form. Theorem 13 asserts that when the foliation contains a compact leaf, then it is itself a fibration over a circle. 
We outline an alternative proof of Theorem 13 that does not use Reeb's theorem (or rather, does not use the aforementioned key point of its proof):

Proposition 15. Let $\mathcal{F}$ be a transversely orientable codimension one foliation of a connected manifold $M$ with $c_{\mathcal{F}}=0$. Then:

(1) there exists a nontrivial family of diffeomorphisms $\Phi_{t}: M \rightarrow$ $M$, defined for $t \in(-\varepsilon, \varepsilon)$, that takes leaves to leaves;

(2) if $\mathcal{F}$ contains a compact leaf $L$, then all leaves are compact;

(3) and furthermore there exists a saturated neighbourhood $\mathcal{U}$ of $L$ and a projection $f: \mathcal{U} \rightarrow I$ such that the foliation is diffeomorphic to the foliation given by the fibers of $p$.

Proof. (1) Let $\alpha$ be a closed defining one-form of the foliation $\mathcal{F}$, and $v$ a vector field on $M$ such that $\alpha(v)=1$; this vector field is transversal to the foliation $\mathcal{F}$. The flow $\Phi_{t}$ of the vector field $v$, defined for $t \in(-\varepsilon, \varepsilon)$ for small enough $\varepsilon$, takes leaves to leaves diffeomorphically, since

$$
\mathcal{L}_{v} \alpha=\iota_{v} d \alpha+d \iota_{v} \alpha=0
$$

(2) Let $N$ be the union of all compact leaves in $M$. The set $N$ is open: Given a leaf $L$ contained in $N$, the set

$$
\left\{\Phi_{t}(L) \mid t \in(-\varepsilon, \varepsilon)\right\}
$$

is an open neighborhood of $L$ contained in $N$.

To see that $M \backslash N$ is open, let $L^{\prime}$ be a leaf in $M \backslash N$ and $m \in$ $L^{\prime}$ a point. There exists a neighborhood $V$ of $m$ in $L^{\prime}$ and an $\varepsilon>0$ such that $\Phi_{t}\left(L^{\prime}\right)$ is well defined for $t \in(-\varepsilon, \varepsilon)$, let $U=\left\{\Phi_{t}\left(L^{\prime}\right): t \in(-\varepsilon, \varepsilon)\right\}$ be a neighborhood of $m$ in $M$. If $m$ is not an interior point of $M \backslash N$, there exists a compact orbit $L$ which intersects $U$, i.e., there would exist $m^{\prime} \in V$ and $t_{0} \in$ $(-\varepsilon, \varepsilon)$ such that $\Phi_{t_{0}}\left(m^{\prime}\right) \in L$. But this implies that $\Phi_{-t_{0}}(L)$ intersects $L^{\prime}$, and hence that $\Phi_{-t_{0}}(L) \subset L^{\prime}$, and furthermore that $\Phi_{-t_{0}}(L)=L^{\prime}$, so $L^{\prime}$ would be compact, which contradicts the original assumption.

Since $N$ is nonempty and $M$ is connected, we must have $N=M$.

(3) Because $i_{L}^{*} \alpha=0$ with $L$ compact, Poincaré lemma guarantees the existence of a tubular neighbourhood $\mathcal{U}$ of $L$ and function $f$ on it such that the leaf $L$ is the zero level set of $f$ and $\alpha=d f$ on $\mathcal{U}$. Shrinking $\mathcal{U}$ as necessary, we can assume it is a saturated neighbourhood and that the leaves are level sets of $f$. 
So far we have proved that foliations with vanishing first invariant $c_{\mathcal{F}}$ are locally trivial fibrations in the neighbourhood of a given compact leaf. When the manifold is compact, we can use the transverse vector field $v$ of the proof of (11) in Proposition 15 to drag this fiber bundle structure, and by a Cĕch-type construction obtain a global fiber bundle over $\mathbb{S}^{1}$. Furthermore, the choice of a transverse vector field $v$ whose flow takes leaves to leaves gives us an Ehresmann connection, which we use to lift the closed loop on the base of the fibration and thus obtain a holonomy map $\phi: L \rightarrow L$, thus obtaining the following:

Corollary 16. Let $\mathcal{F}$ be a transversely orientable codimension one foliation of a compact connected manifold $M$ with $c_{\mathcal{F}}=0$, and assume that the foliation contains a compact leaf $L$. Then, the manifold $M$ is the mapping toru 4 of the diffeomorphism $\phi: L \rightarrow L$ given by the holonomy map of the fibration over $\mathbb{S}^{1}$.

Remark 17. If the foliation $\mathcal{F}$ satisfies $c_{\mathcal{F}}=0$ but has no compact leaves, and instead there exists a leaf $L$ such that $H^{1}(L, \mathbb{R})=0$ (hypothesis of Thurston's theorem [Th]), then it can be proved that the foliation is a fibration over $\mathbb{S}^{1}$.

3.3. Vanishing (first and) second invariant(s): a Poisson result. We want to look in this section at the case of vanishing first and second invariants, and for that we must now assume that $M$ is endowed with a regular corank one Poisson structure and that $\mathcal{F}$ is the corresponding symplectic foliation. Throughout this section, we will assume that the manifold $M$ is compact and that there exists a compact leaf $L$ (and therefore by Theorem 19 all leaves are compact).

Proposition 18. The two invariants $c_{\mathcal{F}}$ and $\sigma_{\mathcal{F}}$ vanish if and only if there exists a Poisson vector field transversal to the foliation.

Proof. Given $\alpha$ and $\omega$ defining one- and two-forms respectively, let $v$ be the vector field uniquely defined by

$$
\alpha(v)=1 \text { and } \iota_{v} \omega=0 .
$$

Conversely, given a vector field $v$ on $M$ transversal to the foliation $\mathcal{F}$, these equalities uniquely give us defining one- and two-forms $\alpha$ and $\omega$. We must prove that $v$ is Poisson if and only if only if $d \alpha=d \omega=0$, i.e., $c_{\mathcal{F}}=\sigma_{\mathcal{F}}=0$.

\footnotetext{
${ }^{4}$ The mapping torus of $\phi: L \rightarrow L$ is the space $\frac{L \times[0,1]}{(x, 0) \sim(\phi(x), 1)}$.
} 
First, let us assume that $d \alpha=d \omega=0$. Then by (6), we have $\mathcal{L}_{v} \alpha=\mathcal{L}_{v} \omega=0$, and we want to show that $\mathcal{L}_{v} \Pi=0$, where $\Pi$ is the Poisson bivector field on $M$.

Because $\alpha$ and $\omega$ are the defining one- and two- forms of the symplectic foliation induced by $\Pi$ we have,

$$
\iota_{\Pi}\left(\alpha \wedge \omega^{n}\right)=n \alpha \wedge \omega^{n-1} .
$$

Applying $\mathcal{L}_{v}$ to both sides and using $\mathcal{L}_{v} \alpha=\mathcal{L}_{v} \omega=0$ we obtain

$$
\iota_{\left(\mathcal{L}_{v} \Pi\right)}\left(\alpha \wedge \omega^{n}\right)=0,
$$

which implies that $\mathcal{L}_{v} \Pi=0$ because $\alpha \wedge \omega^{n}$ is a volume form.

Now let us assume that $v$ is a Poisson vector field, we will first check that $d \alpha=0$. For this we just need to apply $d \alpha$ to any arbitrary pair of vector fields. Since $\alpha$ is the defining one-form of the symplectic foliation, the two-form $d \alpha$ must vanish on pairs of the form $\left(u_{f}, u_{g}\right)$. For pairs of the form $\left(v, u_{f}\right)$, we have

$$
d \alpha\left(v, u_{f}\right)=v\left(\alpha\left(u_{f}\right)\right)-u_{f}(\alpha(v))-\alpha\left(\left[v, u_{f}\right]\right) .
$$

All three terms on the right vanish, the last one because $v$ being a Poisson vector field implies that $\left[v, u_{f}\right]=u_{v(f)}$. Thus, (7) becomes $d \alpha\left(v, u_{f}\right)=0$, and so $d \alpha=0$.

To see that $d \omega=0$, it suffices to check it on triples of the form $\left(v, u_{f}, u_{g}\right)$. Using $\omega\left(u_{h_{1}}, u_{h_{2}}\right)=\left\{h_{1}, h_{2}\right\}$ and the classical formula

$$
\begin{aligned}
d \omega(u, v, w) & =u(\omega(v, w))+v(\omega(w, u))+w(\omega(u, v))- \\
& -\omega([u, v], w)-\omega([v, w], u)-\omega([w, u], v),
\end{aligned}
$$

we obtain

$$
d \omega\left(v, u_{f}, u_{g}\right)=v(\{f, g\})-\{v(f), g\}-\{f, v(g)\},
$$

which again vanishes when $v$ is a Poisson vector field.

As before, the choice of a transverse vector field $v$ gives an Ehresmann connection on the fibration that we can use to lift the closed loop on the base of the fibration to obtain a holonomy map $\phi: L \rightarrow L$.

Because $v$ is a Poisson vector field, its flow drags the symplectic structure of one leaf to define the Poisson structure on $M$ (because $M$ is a symplectic fibration over a one dimensional base, the GuilleminLerman-Sternberg condition GLS guarantees that the minimal coupling structure yields a unique Poisson structure on $M$ ). Furthermore, 
the parallel transport of the connection preserves the symplectic structure on the leaves, and in particular the holonomy map $\phi$ is a symplectomorphism:

Theorem 19. Let $M$ be an oriented compact connected regular Poisson manifold of corank one and $\mathcal{F}$ its symplectic foliation. If $c_{\mathcal{F}}=\sigma_{\mathcal{F}}=0$ and $\mathcal{F}$ contains a compact leaf $L$, then every leaf of $\mathcal{F}$ is symplectomorphic to $L$.

Furthermore, $M$ is the total space of a fibration over $\mathbb{S}^{1}$ and it is the mapping torus of the symplectomorphism $\phi: L \rightarrow L$ given by the holonomy map of the fibration over $\mathbb{S}^{1}$.

Regular corank one Poisson manifolds with $c_{\mathcal{F}}=\sigma_{\mathcal{F}}=0$ are interesting in Poisson geometry because they can be characterized as manifolds which are the critical hypersurfaces of Poisson $b$-manifolds:

Definition 20. An oriented Poisson manifold $\left(M^{2 n}, \Pi\right)$ is a Poisson $b$-manifold if the map

$$
x \in M \mapsto(\Pi(x))^{n} \in \Lambda^{2 n}(T M)
$$

is transverse to the zero section. In particular, this implies that the critical set $\left\{x \in M \mid(\Pi(x))^{n}=0\right\}$ is a hypersurface.

Theorem 21. GMP Let $(M, \Pi)$ be an oriented compact regular Poisson manifold of corank one, $\mathcal{F}$ its symplectic foliation and $v$ a Poisson vector field transversal to $\mathcal{F}$.

If $c_{\mathcal{F}}=\sigma_{\mathcal{F}}=0$, then there exists a Poisson b-manifold $(U, \tilde{\Pi})$, with $M \subset U$ its critical locus, and such that $\tilde{\Pi}$ restricted to $M$ is $\Pi$. Furthermore, this extension is unique up to isomorphism among the extensions for which $[v]$ is the image of the modular class under the map

$$
H_{\text {Poisson }}^{1}(U) \longrightarrow H_{\text {Poisson }}^{1}(M) \text {. }
$$

Conversely, we will see in GMP that the critical hypersurface of a Poisson b-manifold, endowed with the restriction of the Poisson $b$ structure, is always a regular corank one Poisson manifold with vanishing first and second invariants. Note that in Theorem 21 we do not require that $M$ be foliated by compact leaves.

Example 22. Take $\left(N^{2 n+1}, \pi\right)$ to be a regular corank one Poisson manifold with compact leaves, and let $X$ be a Poisson vector field on it. Consider the product $\mathbb{S}^{1} \times N$ endowed with the bivector field

$$
\Pi=f(\theta) \frac{\partial}{\partial \theta} \wedge X+\pi .
$$


This is a Poisson $b$-manifold provided that $f$ vanishes linearly and the vector field $X$ is transverse to the symplectic leaves of $N$.

The critical locus of this Poisson $b$-structure has as many copies of $N$ as $f$ has zeros. Because of Theorem 10 , the fact that $X$ is a Poisson vector field transverse to the symplectic foliation implies that the two invariants of $N$ vanish. by Theorem 19, each copy of $N$ is a symplectic mapping torus and the gluing diffeomorphism is the time-one map of the flow of $X$.

Example 23. Let $\mathfrak{g}$ be the Lie algebra of the affine group in dimension 2. This is a model for noncommutative Lie algebras in dimension 2 and for a basis $e_{1}, e_{2}$, the bracket is $\left[e_{1}, e_{2}\right]=e_{2}$. From this Lie algebra structure we get the Poisson bivector field

$$
\Pi=y \frac{\partial}{\partial x} \wedge \frac{\partial}{\partial y} .
$$

In this example the critical locus is the $x$-axis, which is non-compact, and the its symplectic leaves are the points of that line. The upper and lower half-planes are open dense symplectic leaves. As we will see in GMP, this is a prototypical example of Poisson $b$-manifolds.

Example 24. Let $M=\mathbb{T}^{3}$ with coordinates $\theta_{1}, \theta_{2}, \theta_{3}$ (we work in a covering) and $\mathcal{F}$ the codimension one foliation with leaves given by

$$
\theta_{3}=a \theta_{1}+b \theta_{2}+k, \quad k \in \mathbb{R},
$$

where $a, b, 1 \in \mathbb{R}$ are fixed and independent over $\mathbb{Q}$. This implies that each leaf is diffeomorphic to $\mathbb{R}^{2}$ [Ma]. Then,

$$
\alpha=\frac{a}{a^{2}+b^{2}+1} d \theta_{1}+\frac{b}{a^{2}+b^{2}+1} d \theta_{2}-\frac{1}{a^{2}+b^{2}+1} d \theta_{3}
$$

is a defining one-form for $\mathcal{F}$ and there is a Poisson structure $\Pi$ on $M$ of which

$$
\omega=d \theta_{1} \wedge d \theta_{2}+b d \theta_{1} \wedge d \theta_{3}-a d \theta_{2} \wedge d \theta_{3}
$$

is the defining two-form. Note that $\alpha$ and $\omega$ are closed, and so the invariants $c_{\mathcal{F}}$ and $\sigma_{\mathcal{F}}$ vanish. Note that in this example leaves are non-compact so Theorem 19 does not apply. However, Theorem 21 still holds, and indeed $(M, \Pi)$ can be extended to $(U, \tilde{\Pi})$ where $U=$ $M \times(-\varepsilon, \varepsilon)$ and $\tilde{\Pi}$ is the bivector field dual to the two-form

$$
\tilde{\omega}=d(\log t) \wedge \alpha+\omega
$$


3.4. Vanishing (first and) second invariants: explicit integration of the Poisson structure. A Lie algebroid structure over a manifold $M$ consists of a vector bundle $E$ together with a bundle map $\rho: E \rightarrow T M$ and a Lie bracket $[,]_{E}$ on the space of sections satisfying, for all $\alpha, \beta \in \Gamma(E)$ and all $f \in C^{\infty}(M)$, the Leibniz identity:

$$
[\alpha, f \beta]_{E}=f[\alpha, \beta]_{E}+L_{\rho(\alpha)}(f) \beta .
$$

Given a Lie groupoid one can naturally associate to it a Lie algebroid structure, but the converse is not true: given a Lie algebroid it is not always possible to produce a smooth Lie groupoid with the prescribed Lie algebroid structure. However, when the Lie algebroid is in fact a Lie algebra (case of Lie algebroid over a point), the integration to a Lie group is guaranteed by Lie's third theorem. In $[\mathrm{CF}$, Marius Crainic and Rui Loja Fernandes solved the problem of integrability of a Lie algebroid to a Lie groupoid.

Given a Poisson manifold $(M, \pi)$, there exists a natural Lie algebroid structure on $T^{*} M$ : the Poisson cotangent Lie algebroid has anchor map $\pi^{\sharp}$ and Lie bracket defined by

$$
[\alpha, \beta]=L_{\pi^{\sharp}(\alpha)}(\beta)-L_{\pi^{\sharp}(\beta)}(\alpha)-d(\pi(\alpha, \beta)) .
$$

In this case the integrability problem consists of associating a symplectic groupoid to this Lie algebroid structure, as studied by Marius Crainic and Rui Loja Fernandes in [CF04. The canonical integration, or Weinstein's groupoid, is a symplectic groupoid integrating the algebroid which is source simply connected.

Recall the following theorem [CF04, Corollary 14],

Theorem 25. [Crainic-Fernandes] Let $M$ be a regular Poisson manifold. Then:

(1) If $M$ admits a leafwise symplectic embedding then every leaf of $M$ is a Lie-Dirac submanifold.

(2) If every leaf of $M$ is a Lie-Dirac submanifold then $M$ is integrable.

The second obstruction class $\sigma_{\mathcal{F}}$ can be interpreted via Gotay's embedding theorem [G0] and measures the obstruction for a closed twoform to exist on $Z$ which restricts to $\omega_{L}$ on each leaf $L$. If $c_{\mathcal{F}}=0$, we get a leafwise symplectic embedding and Theorem 25 guarantees integrability. Furthermore, using Theorem 19 we can obtain an explicit characterization of the Weinstein's integrating groupoid: 
Corollary 26. The Poisson structure on a compact manifold with vanishing invariants $c_{\mathcal{F}}$ and $\sigma_{\mathcal{F}}$ and a compact leaf is integrable (in the Crainic-Fernandes sense) and the Weinstein's symplectic groupoid is a mapping torus.

Proof. Fix a compact symplectic leaf $L$. Consider the Weinstein's groupoid of a symplectic leaf $\left(\Pi_{1}(L), \Omega\right)$ and the product $\left(\Pi_{1}(L) \times\right.$ $\left.T^{*}(\mathbb{R}), \Omega+d \lambda_{\text {liouville }}\right)$ with the product groupoid structure. Let $f$ be the time- 1 flow of $v$, the Poisson vector field transverse to the symplectic foliation. By the construction provided in Theorem 19, $v$ has periodic orbits. Now let $\tilde{f}$ be the lift to $\Pi_{1}(L)$, the Weinstein's groupoid of $L$ and on this product define the "natural lift" of $\tilde{f}$, namely the map $\phi:(\tilde{x},(t, \eta)) \mapsto(\tilde{f},(t+1, \eta))$. This map preserves the product symplectic groupoid structure and thus by identification induces a symplectic groupoid structure on the mapping torus which has $\phi$ as gluing diffeomorphism.

\section{REFERENCES}

[B] M. Bertelson, Foliations associated to regular Poisson structures. Commun. Contemp. Math. 3 (2001), no. 3, 441-456.

[CN] C. Camacho and A. Lins Neto, Geometric theory of foliations, Birkhauser (1985).

[CF04] M. Crainic and R.L. Fernandes, Integrability of Poisson brackets, J. of Differential Geometry 66, (2004) pp. 71-137.

[CF] M. Crainic and R.L. Fernandes, Integrability of Lie brackets, Annals of Mathematics 157, (2003) pp. 575-620.

[ELW] S. Evens, J-H. Lu, A. Weinstein, Transverse measures, the modular class and a cohomology pairing for Lie algebroids. Quart. J. Math. Oxford Ser. (2) 50 (1999), no. 200, 417-436.

[Go] M. Gotay, On coisotropic embeddings of presymplectic manifolds, Proc. Amer. Math. Soc. 84 (1982), pp. 111-114.

[GLSW] M. Gotay, R.Lashof, J. Sniaticky, A. Weinstein, Closed forms on symplectic fibre bundles, Comment. Math. Helvetici 58 (1983), pp. 617-621 .

[GLS] V. Guillemin, E. Lerman and S. Sternberg, Symplectic fibrations and multiplicity diagrams, Cambridge University Press (1996).

[GMP] V. Guillemin, E. Miranda and A.R. Pires, Symplectic and Poisson geometry of b-manifolds, preprint, (2010).

[KLW] Y. Kosmann-Schwarzbach, C. Laurent-Gengoux and A. Weinstein Modular classes of Lie algebroid morphisms. Transform. Groups 13 (2008), no. 3-4, pp. 727-755.

[Ko] Y. Kosmann-Schwarzbach, Poisson manifolds, Lie algebroids, modular classes: a survey, SIGMA, 4 (2008), 005, 30 pp.

[Li] H. Li, Topology of co-symplectic/co-kahler manifolds, Asian. J. Math, Vol $12, \mathrm{n} 4,(2008)$ p 527-544. 
[L] P. Libermann, Sur les automorphismes infinitésimaux des structures symplectiques et des structures de contact. 1959 Colloque Géom. Diff. Globale (Bruxelles, 1958) pp. 37-359 Centre Belge Rech. Math., Louvain.

[Ma] V. Mamaev, Codimension one foliations of flat 3-manifolds, Sbornik: Mathematics 187:6 (1996), pp. 823-833.

[Me] R. Melrose, Atiyah-Patodi-Singer Index Theorem, A.K. Peters, Wellesley (1993).

[Re] G. Reeb, Sur certaines propriétés toplogiques des variétés feuillétées, Actualités Sci. Indust., 1183 (1952), Paris: Hermann.

[Th] W.P. Thurston, Existence of codimension-one foliations. Ann. of Math. (2) 104 (1976), no. 2, pp. 249-268.

[Ti] D. Tischler, On fibering certain foliated manifolds over $\mathbb{S}^{1}$, Topology, Vol 9 (1970), pp. 153-154.

[We] A. Weinstein, The modular automorphism group of a Poisson manifold, J. Geom. and Phys. 23 (1997), no. 3-4, pp. 379-394.

Victor Guillemin, Department of Mathematics, Massachussets InStitute of Technology, CAMBridge MA, US, e-mail: vwg@math.mit.edu

Eva Miranda, Departament de Matemàtica Aplicada I, Universitat Politècnica de Catalunya, Barcelona, Spain, e-mail: eva.miranda@upc.edu

Ana Rita Pires, Department of Mathematics, Massachussets Institute of Technology, CAmbridge MA, US, e-mail: arita@math.mit.edu 